\title{
A Novel Rodent Model That Mimics the Metabolic Sequelae of Obese Craniopharyngioma Patients
}

\author{
CHRISTIAN L. ROTH, JAMES E. BLEVINS, MELISSA RALSTON, CLINTON ELFERS, KAYOKO OGIMOTO, KARL J. KAIYALA, \\ AND GREGORY J. MORTON
}

\begin{abstract}
Seattle Children's Research Institute [C.L.R., M.R., C.E.], University of Washington, Seattle, Washington 98101; Division of Metabolism, Endocrinology and Nutrition [J.E.B.], Seattle VA Puget Sound Health Care System, Seattle, Washington 98108; Department of Medicine [K.O., G.J.M.], University of Washington, Seattle, Washington 98109; Department of Dental Public Health Sciences [K.J.K.], University of Washington, Seattle, Washington 98195
\end{abstract}

\begin{abstract}
Patients with craniopharyngioma (CP), a tumor located in the pituitary and/or hypothalamus, are susceptible to developing obesity and many metabolic complications. The study aim was to create a rodent model that mimics the complex neuroanatomical and metabolic disturbances commonly seen in obese CP patients. We compared the metabolic phenotype of animals with three distinct types of hypothalamic lesions: 1) destruction of the arcuate nucleus (ARC) induced by monosodium glutamate (MSG), 2) electrolytic lesion of the adjacent ventromedial nucleus (VMN) alone, 3) both the VMN and dorsomedial nucleus (DMN), or a 4) combined medial hypothalamic lesion (CMHL) affecting the VMN, DMN, and the ARC. Only the CMHL model exhibited all key features observed in patients with hypothalamic obesity induced by CP. These features included excessive weight gain due to increased adiposity, increased food intake, and pronounced hyperinsulinemia and hyperleptinemia. Similar to characteristics of patients with CP, CMHL animals exhibited reduced plasma levels of alpha-melanocyte stimulating hormone and reduced ambulatory activity compared with weight-matched controls. Therefore, the CMHL model best mimics the complex metabolic abnormalities observed in obese $\mathrm{CP}$ patients compared with lesions to other hypothalamic areas and provides a foundation for future pharmacological approaches to treat obesity in children with hypothalamic damage. (Pediatr Res 69: 230-236, 2011)
\end{abstract}

$\mathrm{C}$ raniopharyngioma $(\mathrm{CP})$ is an embryological tumor with low-grade histological malignancy originating from ectodermic cell remnants of the Rathke pouch and developing along the hypothalamic-pituitary region $(1,2)$. Tumor resection, with or without radiotherapy, causes obesity in approximately one half of patients with $\mathrm{CP}$ and is characterized by increased food intake, reduced physical activity, and daytime sleepiness (3-7). Moreover, thus far, dietary or pharmaceutical approaches used to treat obesity in patients with CP after surgery have been largely unsuccessful or had limited success $(8-13)$.

\footnotetext{
Received August 17, 2010; accepted October 6, 2010.

Correspondence: Christian L. Roth, M.D., Division of Endocrinology, Seattle Children's Hospital Research Institute, 1900 Ninth Avenue, Seattle, WA 98101; e-mail: christian.roth@seattlechildrens.org

Supported by the Nutrition Obesity Research Center (NORC, DK035816) at the University of Washington and by an American Heart Association Scientist Development Grant [G.J.M.].

Supplemental digital content is available for this article. Direct URL citations appear in the printed text and are provided in the HTML and PDF versions of this article on the journal's Web site (www.pedresearch.org).
}

The hypothalamus is well recognized as a key brain area critical in the regulation of energy balance. Based on brain lesion experiments, the ventromedial nucleus (VMN) and the paraventricular nucleus (PVN) of the hypothalamus were identified as "satiety" centers, as lesions to these brain areas caused hyperphagia and obesity, while electrolytic stimulation of either the VMN or PVN reduced food intake (14). Conversely, the lateral hypothalamic area (LHA) was identified as a "hunger" center, as stimulation of this brain area increased feeding while lesioning of the LHA caused reduced food intake and weight loss $(15,16)$. In addition, the arcuate nucleus (ARC), which is located adjacent to the floor of the third ventricle, senses and responds to signals that convey information regarding both energy stores and nutrient availability and thereby makes adjustments in systems that control food intake and energy expenditure (EE) (17).

The aim of this study was therefore to create a rat model that effectively mimics the neuroanatomical and metabolic disturbances characteristic of obese $\mathrm{CP}$ patients, so as to guide translational research aimed at the identification of options to prevent or treat $\mathrm{CP}$-induced obesity in humans. Our data suggest that compared with lesions to the ARC, VMN, and dorsomedial nucleus (DMN), a combined medial hypothalamic lesion (CMHL) that affects each of these brain areas causes an array of metabolic abnormalities resembling those observed in obese CP patients and may therefore help to guide future investigation into the efficacy of pharmacotherapy of obesity in patients with $\mathrm{CP}$.

\section{METHODS}

Animals. Pregnant female Sprague-Dawley rats were purchased from Charles River Laboratory (Wilmington, MA). Litter sizes were adjusted to 10-11 animals per dam. Newborn male pups were treated with monosodium glutamate (MSG) or saline as described below. After weaning at $22 \mathrm{~d}$ of age, pups were raised in groups of 3-4 animals per cage. For stereotaxic electrolytic lesions, young adult male Sprague-Dawley rats (Charles River), weighing 210-230 g, were used. Adult male obese $\left(f a^{k} / f a^{k}\right)$ Koletsky rats (Vassar

\footnotetext{
Abbreviations: ARC, arcuate nucleus; CP, craniopharyngioma; CMHL, combined medial hypothalamic lesions; DMN, dorsomedial nucleus; LBM, lean body mass; LHA, lateral hypothalamic area; L-MSG, L-monosodium glutamate; MC4R, melanocortin-4 receptor, $\boldsymbol{\alpha}$-MSH, alpha melanocyte-stimulating hormone; NPY, neuropeptide Y; PVN, paraventricular nucleus; VMN, ventromedial nucleus
} 

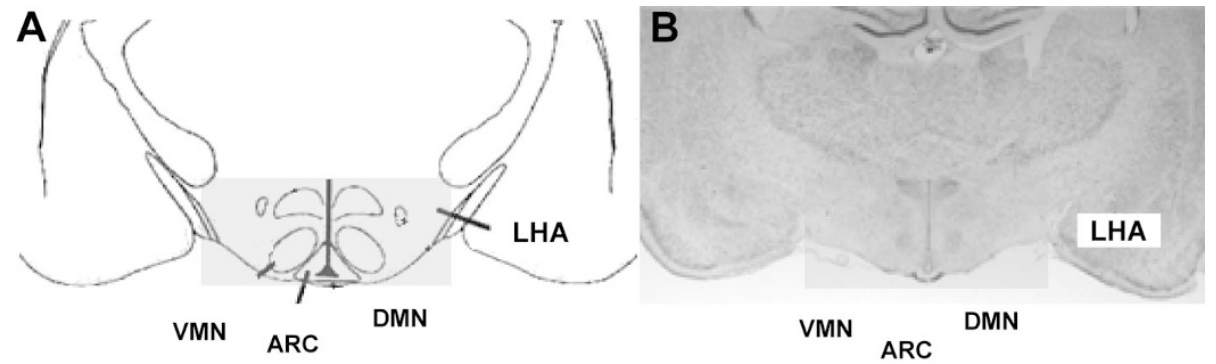

Figure 1. (A) Schematic overview of the mediobasal hypothalamus and placement of electrodes to target the VMN. (B) Representative coronal brain sections $(-2.6 \mathrm{~mm}$ from bregma) from a sham-treated control animal; electrolytic lesions to the $\mathrm{VMN}(C), \mathrm{VMN} / \mathrm{DMN}(D)$, or a CMHL (ARC, VMN, and DMN, $(E)$ ).
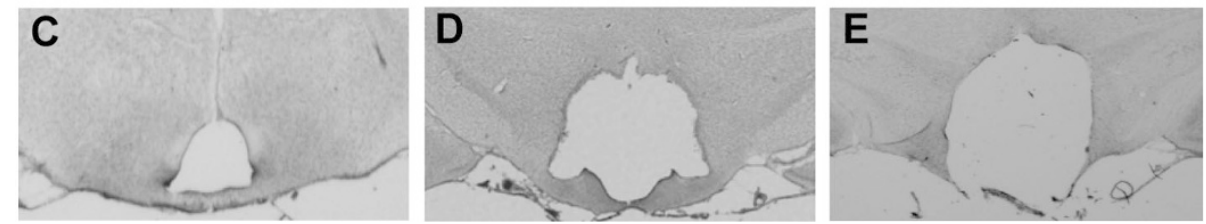

College, Poughkeepsie, NY) were generated from serial backcrosses (N10 equivalent) of the $f a^{k}$ mutation to the inbred rat strain, LA/N. Animals were maintained on a 12/12-h light-dark cycle (07:00-19:00 h) in a temperature and humidity controlled room with ad libitum access to regular chow (5053 Pico Lab Rodent Diet 20; Purina LabDiet, Richmond, CA) and water. All procedures performed were approved by the Institutional Animal Care and Use Committee at the Seattle Children's Research Institute and were in accordance with the National Institutes of Health Guide for the Care and Use of Laboratory Animals.

MSG-induced lesions of the ARC. During the first $10 \mathrm{~d}$ of life, male pups ( $n=8-10 /$ group) received a $3.5 \mathrm{mg} / \mathrm{g}$ body mass MSG (L-MSG; Sigma Chemical Co-Aldrich, St. Louis, MO) or $0.9 \%$ saline s.c. with the final injection volume adjusted with saline to $25 \mu \mathrm{L}$ per rat. Pups received injections every other day for a total of five doses $(18,19)$. Body weight and food intake measures were recorded weekly.

Electrolytic lesions. This study used the coordinate system to determine the placement of electrode using the interaural line and bregma as a reference. Animals were mounted in a Kopf stereotaxic instrument (Tujunga, CA) under anesthesia with isoflurane-oxygen mixture. The ratio of isoflurane-oxygen was adjusted to $2-4 \%$ throughout the surgical procedure. The upper incisor bar was $3.5 \mathrm{~mm}$ above the interaural line. To target lesions to specific hypothalamic nuclei, we placed the insulated stainless steel electrode to stereotaxic coordinates based on previously published studies (20-22). Specifically, to target lesions to the VMN ( $n=6-8$ group), an electrical current was passed through the tip of the electrode placed $2.6 \mathrm{~mm}$ posterior to the bregma, $0.7 \mathrm{~mm}$ lateral to the midsagittal line, and $9.6 \mathrm{~mm}$ ventral from the skull according to the atlas of Paxinos and Watson (23). An electrical current of $1.2 \mathrm{~mA}$ was then passed for $15 \mathrm{~s}$ on each side (24). To create a lesion to the VMN and DMN, the electrode was stereotaxically placed to the same position and the electrical current was increased to $2.0 \mathrm{~mA}$ for $15 \mathrm{~s}$ on both sides (Fig. $1 C$ and $D$ ). To target the ARC, in addition to the VMN and DMN, a novel combined ARC/VMN/DMN (CMHL, see Fig. $1 E$ ) lesion was created by combining two lesions on each side. First, a VMN/DMN lesion was been created [using the coordinates $2.6 \mathrm{~mm}$ posterior to the bregma, $0.5 \mathrm{~mm}$ lateral to the midsagittal line, and $8.6 \mathrm{~mm}$ ventral from the skull $(2.0 \mathrm{~mA}$ for $15 \mathrm{~s}$ on each side)]. Using the same lateral and posterior coordinates, the electrode was lowered to $10.0 \mathrm{~mm}$ ventral from the skull, which not only results in a lesion of the ARC but also the ventral part of the VMN (ARC/VMN, $2.0 \mathrm{~mA}$ for $15 \mathrm{~s}$ on each side). Animals were allowed to recover and food intake and body weight were measured weekly.

Meal pattern, EE, and body composition analysis. Body composition analysis and indirect calorimetry and locomotor activity measurements were performed using the University of Washington's Nutrition Obesity Research Center Animal Studies Core facility. EE was assessed using a computercontrolled calorimetry system (Oxymax; Columbus Instruments, $\mathrm{OH}$ ) as previously described (25) and analyzed using a regression analysis approach (26). Locomotor activity was assessed by the infrared beam breaks using an Opto-Varimetrix-3 sensor system (Columbus Instruments, Columbus, $\mathrm{OH}$ ). Body composition analysis of lean mass, fat mass, and water content in vivo was performed in immobilized rats by quantitative magnetic resonance (EchoMRI body composition analyzer; Echo Medical Systems, Houston, TX).

Collection of tissues and fluids. Blood was collected from tail vein or by collection of trunk blood at the end of the experiments. Blood was drawn into prechilled EDTA tubes, centrifuged immediately at $4^{\circ} \mathrm{C}$, aliquoted, and stored at $-80^{\circ} \mathrm{C}$. Plasma immunoreactive insulin and leptin levels were determined by ELISA (Crystal Chem, Chicago, IL). Plasma levels of corticosterone and testosterone were measured at the University of Virginia Center for Research in Reproduction (Charlottesville, VA). Plasma IGF-1 was determined by ELISA (Quantikine, R\&D systems, Minneapolis, MN), plasma thyroxine (T4) was determined by ELISA (Calbiotech, Spring Valley, CA), and plasma alpha melanocyte-stimulating hormone ( $\alpha$-MSH) levels were determined by a commercially available enzyme immunoassay (EIA) kit (Phoenix Pharmaceuticals, Burlingame, CA) after reversed-phase solid-phase extraction of plasma samples. For all measurements, intra-assay coefficients of variation were $<7 \%$ and interassay coefficients of variation were $<10 \%$.

Immunohistochemical verification of lesion site. At the completion of the study, brains were immediately removed and a 5-mm-thick coronal slice including the hypothalamus was placed into a $20 \mathrm{~mL}$ scintillation vial filled with $4 \%$ paraformaldehyde in PBS (USB, Cleveland, OH) for $48 \mathrm{~h}$, followed by $48 \mathrm{~h}$ in $25 \%$ sucrose in PBS, which served as a cryoprotectant. Brains were then stored at $-80^{\circ} \mathrm{C}$. Coronal sections $(50 \mu \mathrm{m})$ were taken, mounted on microscope slides, and stained with Cresyl violet as previously described (27). Animals with misplaced lesions were excluded from analysis $(<10 \%$ animals). Slides were examined under an Olympus SZX16 microscope using a SDF PLAPO $5 \times$ objective lens.

Statistical analysis. All results are expressed as mean \pm SEM. Statistical analyses were performed using GraphPad Prism Software (LA Jolla, CA) and SPSS (version 17; IBM, Chicago, IL). For unadjusted analyses, a one-way ANOVA with Newman-Keuls Multiple Comparison Test post hoc test was used to compare mean values between multiple groups, and a two-sample unpaired $t$ test was used for two-group comparisons. In analyses requiring adjustment for body size disparity, analysis of covariance using the general linear model procedure in SPSS was used to compute adjusted group means. In all instances, $p<0.05$ was considered significant.

\section{RESULTS}

Effect of MSG-treatment on energy homeostasis and hormonal measures. Lesions selective for the ARC were performed by neonatal application of MSG using a standard dose of $3.5 \mathrm{mg} / \mathrm{g}$ body mass per injection $(18,19)$. At $15 \mathrm{wk}$ of age, MSG-treated rats had a greater percent body fat, despite significant reductions of body weight, food intake, and body length relative to vehicle-treated controls (Table 1). This increase in $\%$ body adiposity was accompanied by an increase in the Lee index, a marker of adiposity calculated by the cube root of body weight $(\mathrm{g}) \times 10$ divided by naso-anal length (mm) (28), and increased plasma levels of insulin and leptin (Table 1). Although there were no differences in plasma corticosterone or testosterone levels, consistent with a deficiency of growth hormone and reduced linear growth, plasma IGF-1 levels were significantly reduced in MSG-treated animals compared with vehicle-treated controls (Table 1).

Effect of electrolytic lesions to the VMN and VMN/DMN on energy homeostasis and hormonal measures. We next performed electrolytic lesions targeted to the VMN. Histolog- 
Table 1. Weight, growth, adiposity, food intake, and hormones in 15-wk-old ARC lesioned male rats after neonatal MSG treatment

\begin{tabular}{lcc}
\hline & Control (saline) & ARC lesion (MSG) \\
\hline Body weight (g) & $381.7 \pm 6.1$ & $282.1 \pm 6.5^{*}$ \\
Length (mm) & $238.4 \pm 1.1$ & $210.6 \pm 2.1 \dagger$ \\
Lee index & $0.303 \pm 0.001$ & $0.310 \pm 0.002^{*}$ \\
Body fat (\%) & $18.5 \pm 1.3$ & $36.5 \pm 0.9 \dagger$ \\
Food intake (age 9 wk) & $17.3 \pm 0.7$ & $14.3 \pm 2.8$ \\
Corticosterone (ng/mL) & $240 \pm 43$ & $353 \pm 86$ \\
Testosterone (ng/mL) & $237 \pm 42$ & $229 \pm 65$ \\
Insulin (ng/mL) & $0.69 \pm 0.3$ & $1.41 \pm 0.2$ \\
Leptin $(\mathrm{ng} / \mathrm{mL})$ & $4.77 \pm 0.6$ & $10.6 \pm 1.5 \ddagger$ \\
IGF-1 $(\mathrm{ng} / \mathrm{mL})$ & $795 \pm 84$ & $461 \pm 71 \ddagger$ \\
\hline
\end{tabular}

Values are represented as mean \pm SEM.

$* p<0.05$.

$\dagger p<0.001$.

$\ddagger p<0.01$.

ical analysis using cresyl violet staining was used to verify the site of the lesion to the VMN relative to sham-treated controls (Fig. 1A-C). As expected, an electrolytic lesion to the VMN caused an increase in body weight gain and transiently increased food intake relative to sham-treated controls, without changes in body length. This increase in adiposity was accompanied by an increase in plasma leptin levels (d 28) relative to sham-treated controls without changes in plasma insulin levels. We next performed an electrolytic lesion targeting both the VMN and DMN. Histological analysis with cresyl violet staining revealed lesions to the VMN and large areas of the DMN, while the ARC remained intact (Fig. 1D). This VMN/ DMN lesion caused a further increase in body weight gain, body adiposity (as measured by the Lee index), and food intake compared with lesions restricted to the VMN alone or sham-treated controls. In addition, plasma insulin and leptin levels were significantly increased in VMN/DMN lesioned rats compared with sham-treated controls, while plasma IGF-1 and testosterone levels were reduced (Table 2; Fig. $2 E$ and $F$ ).

Effect of CMHL on energy homeostasis and hormonal measures. As patients with $\mathrm{CP}$ susceptible to hypothalamic obesity often have damage that includes the hypothalamic
ARC, VMN, and DMN, we created a combined CMHL to mimic this pattern of brain injury (Fig. $1 E$ ). CMHL rats exhibited a greater increase in body weight gain, food intake, and obesity compared with lesions to the VMN or VMN/ DMN, particularly during the first 2 wk after surgery (Table 2 ; Fig. 3). In addition, CMHL rats exhibited marked increases of plasma insulin and leptin levels within $7 \mathrm{~d}$ after surgery (Fig. $4 A$ and $B$ ) and exhibited reduced plasma $\alpha$-MSH levels $7 \mathrm{~d}$ postsurgery relative to sham-treated controls and VMN/DMN lesioned rats (plasma $\alpha$-MSH, CMHL $0.092 \pm 0.015$, CMHL sham $0.140 \pm 0.016, \mathrm{VMN} / \mathrm{DMN} 0.172 \pm 0.019, \mathrm{VMN} /$ DMN sham $0.162 \pm 0.007 \mathrm{ng} / \mathrm{mL} ; p=0.03$ CMHL versus CMHL sham; Fig. S1, http://links.lww.com/PDR/XXX). Although plasma corticosterone and testosterone levels were reduced $7 \mathrm{~d}$ after surgery, these were maintained at levels similar to those of sham-treated controls throughout the experiment, while IGF-1 plasma levels were reduced (Table 2; Fig. $4 C$ and $D$ ). Plasma T4 levels were reduced at all time points after CMHL (Fig. 4E).

Parameters assessed in metabolic cages. To determine whether reduced ambulatory activity and/or metabolic rate also contribute to the phenotype of CMHL rats, we placed both CMHL and sham-treated rats in metabolic cages. We used a regression-based approach, which is now considered the recommended method for normalizing EE in rodents to account for variation in both fat mass and lean body mass (26). To further control for changes in body weight and body fat, we also studied genetically obese, leptin receptor-deficient Koletsky rats. Here, we found that ambulatory activity levels in CMHL rats were reduced relative to Koletsky rats, even after adjusting for differences of body size. After adjusting for lean body mass, fat mass, and activity, EE did not differ between CMHL rats compared with sham-treated controls, although physical activity levels were significantly reduced (Fig. 5A-D). Thus, obesity in this model does not involve a hypometabolic mechanism after correction for the different levels of activity. However, when EE was expressed as a percentage of control, i.e. CMHL normalized to sham-treated rats and obese Ko-

Table 2. Weight, growth, adiposity, food intake, and hormones in 15-wk-old male rats after electrolytic lesion at age of 8 wk

\begin{tabular}{|c|c|c|c|c|c|}
\hline & VMN sham & VMN & VMN/DMN & CHML sham & CMHL \\
\hline Body weight (g) & $363 \pm 6.2$ & $423 \pm 24 *$ & $451 \pm 16 \dagger$ & $392.0 \pm 13.93$ & $465.4 \pm 22.5^{*}$ \\
\hline Length (mm) & $236.2 \pm 2.1$ & $233.0 \pm 3.4$ & $236.8 \pm 2.7$ & $238.8 \pm 2.9$ & $225.4 \pm 1.8 \dagger$ \\
\hline Lee index & $0.301 \pm 0.002$ & $0.320 \pm 0.003 \ddagger$ & $0.322 \pm 0.005 \dagger$ & $0.303 \pm 0.003$ & $0.342 \pm 0.006$ \$ \\
\hline Food intake§ & $19.3 \pm 0.6$ & $24.0 \pm 1.1 \dagger$ & $28.0 \pm 1.9 \dagger$ & $19.4 \pm 0.4$ & $31.9 \pm 2.4 \dagger$ \\
\hline Food intake\| & $19.6 \pm 0.9$ & $21.3 \pm 1.2$ & $24.7 \pm 1.8^{*}$ & $17.7 \pm 0.9$ & $26.3 \pm 2.9^{*}$ \\
\hline Food intakeII & $19.8 \pm 1.4$ & $21.0 \pm 1.5$ & $22.3 \pm 0.9$ & $18.6 \pm 0.8$ & $22.1 \pm 1.6$ \\
\hline Corticosterone $(\mathrm{ng} / \mathrm{mL})$ & $360 \pm 40$ & $263 \pm 38$ & $261 \pm 47$ & $369 \pm 45$ & $344 \pm 47$ \\
\hline Testosterone $(\mathrm{ng} / \mathrm{mL})$ & $383 \pm 57$ & $239 \pm 111$ & $193 \pm 28 *$ & $187 \pm 37$ & $210 \pm 74$ \\
\hline Insulin $(\mathrm{ng} / \mathrm{mL}) \S$ & $0.89 \pm 0.22$ & $1.22 \pm 0.14$ & $1.96 \pm 0.31 *$ & $0.62 \pm 0.18$ & $1.52 \pm 0.46^{*}$ \\
\hline Leptin $(\mathrm{ng} / \mathrm{mL}) \S$ & $3.6 \pm 0.6$ & $8.6 \pm 3.5$ & $9.6 \pm 2.6^{*}$ & $7.3 \pm 1.0$ & $46.8 \pm 8.9 \dagger$ \\
\hline IGF-1 (ng/mL) & $736 \pm 86$ & n.d. & $374 \pm 51 \dagger$ & $921 \pm 17$ & $596 \pm 8 \dagger$ \\
\hline
\end{tabular}

Summarized data from two experiments (experiment 1: sham, VMN, VMN/DMN; experiment 2: sham, CMHL). Values are represented as mean \pm SEM.

$* p<0.05$.

$\dagger p<0.01$.

$\ddagger p<0.001$.

$\S$ Age of 9 wk, 1 wk after lesion.

|l Age of 12 wk, 4 wk after lesion.

II Age of 15 wk, 7 wk after lesion.

n.d., not determined. 

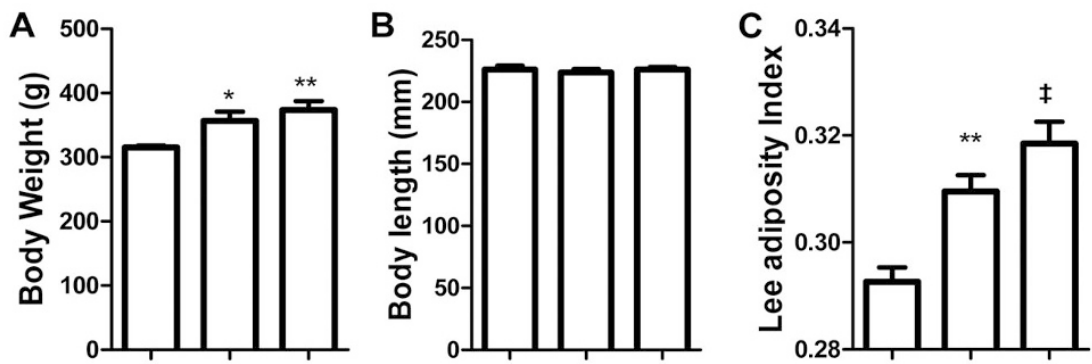

Figure 2. Effects of a lesion to either the VMN, the VMN and DMN relative to shamtreated controls on body weight $(A)$, body length $(B)$, the Lee index $(C)$, food intake $(D)$, plasma insulin $(E)$, and leptin $(F)$ levels at d 28. $* p<$ $0.05, * * p<0.01, \ddagger p<0.001$ vs. sham-treated controls.
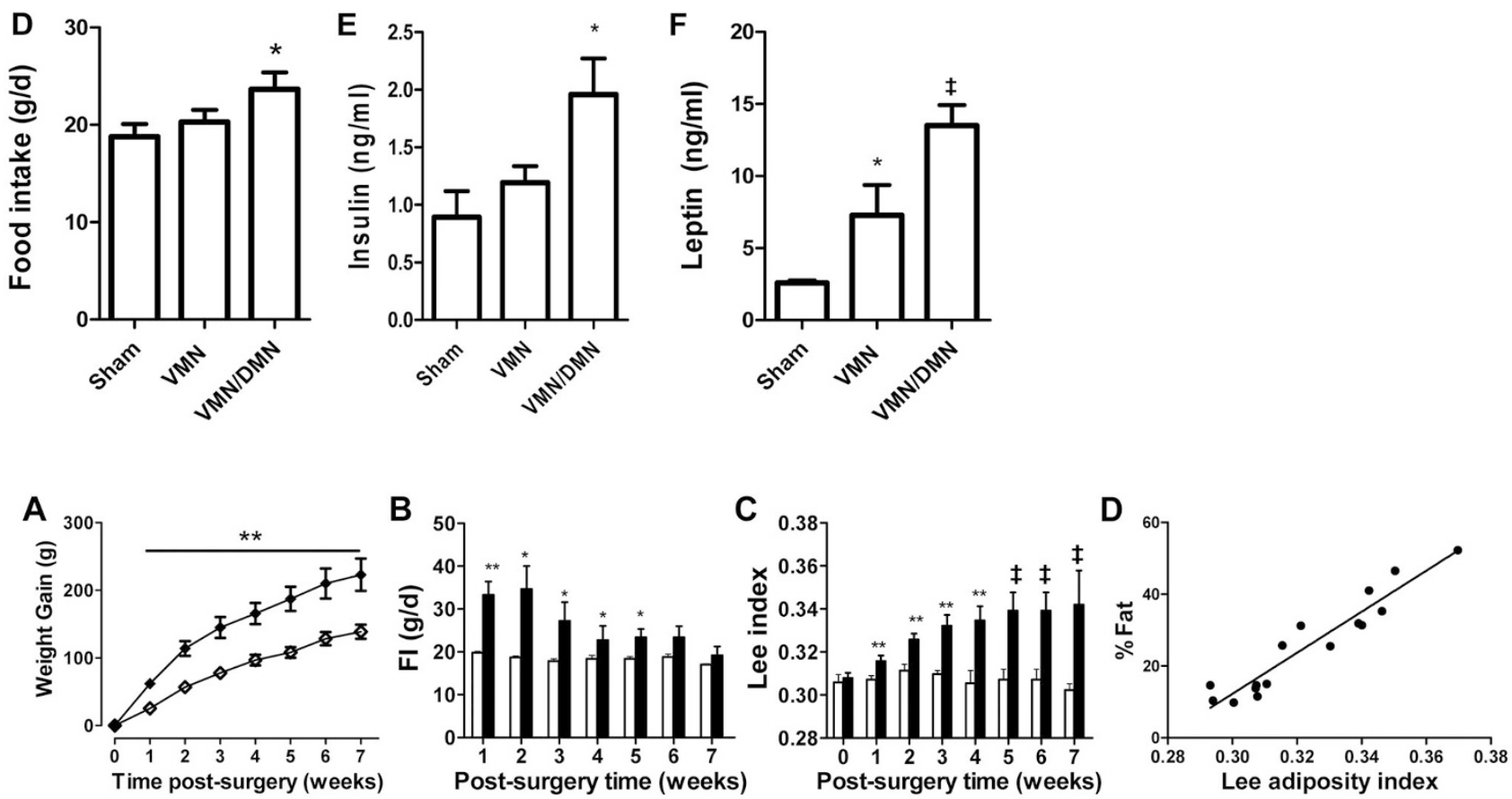

Figure 3. Body weight gain $(A)$, food intake $(B)$, and the Lee adiposity index $(C)$ in CMHL $(\diamond$ and $\mathbf{\square}) v s$. sham-operated rats $(\diamond$ and $\square)$. * $p<0.05$, ** $p<$ $0.01, \ddagger p<0.001 \mathrm{vs}$. sham-treated controls. Correlation between the Lee adiposity index and \% body fat assessed by magnetic resonance in body composition analysis at wk $7\left(r^{2}=0.904, p<0.0001, D\right)$.
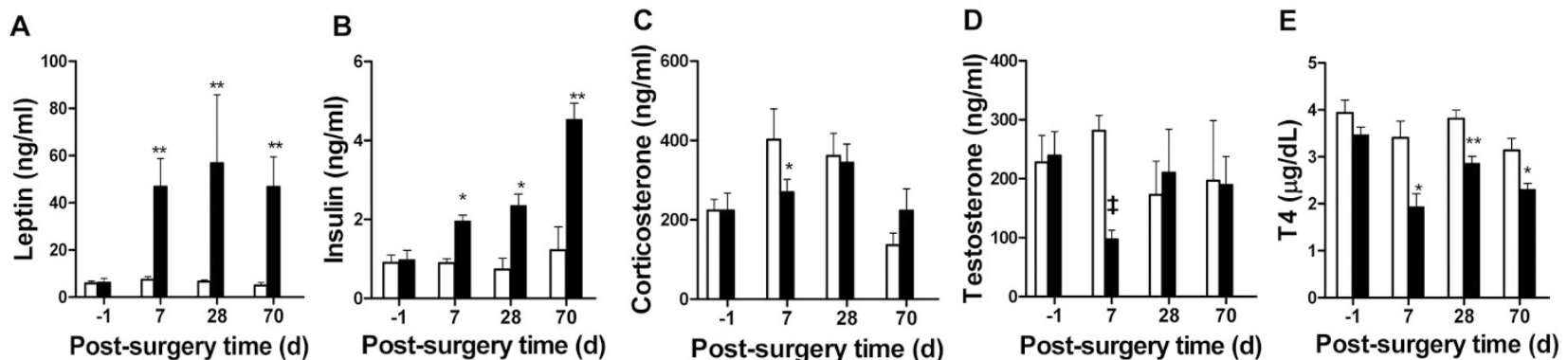

Figure 4. Plasma leptin $(A)$, insulin $(B)$, corticosterone $(C)$, testosterone $(D)$, and T4 levels $(E)$ before and following surgery in CMHL rats $(\square)$ vs. sham-operated rats $(\square)$. $* p<0.05, * * p<0.01, \ddagger p<0.001$ vs. sham-treated controls.

letsky rats to lean Koletsky rats, unadjusted EE was lower in CMHL than in obese Koletsky rats (Fig. $5 E$ ).

\section{DISCUSSION}

Although lesions targeting individual hypothalamic nuclei have been studied for many years in rodents $(14,18,24,29-$ 32 ), these lesion models fail to recapitulate the metabolic sequelae characteristic of obese CP patients. Patients with CP with increased susceptibility to obesity typically have tumors that affect multiple hypothalamic areas that regulate energy balance (33). Although recent studies using gene-specific knockout models were strong to identify key mediators of energy homeostasis and monogenic causes of obesity, these models could not be applied for our purpose, as more complex interacting systems are affected by hypothalamic damage including several neuronal systems and cross-fibers. We therefore created a novel CMHL model in which the ARC, VMN, and DMN are each destroyed bilaterally in an effort to mimic the unique features characteristic of obese CP patients. Body weight gain, body adiposity, and food intake were each in- 

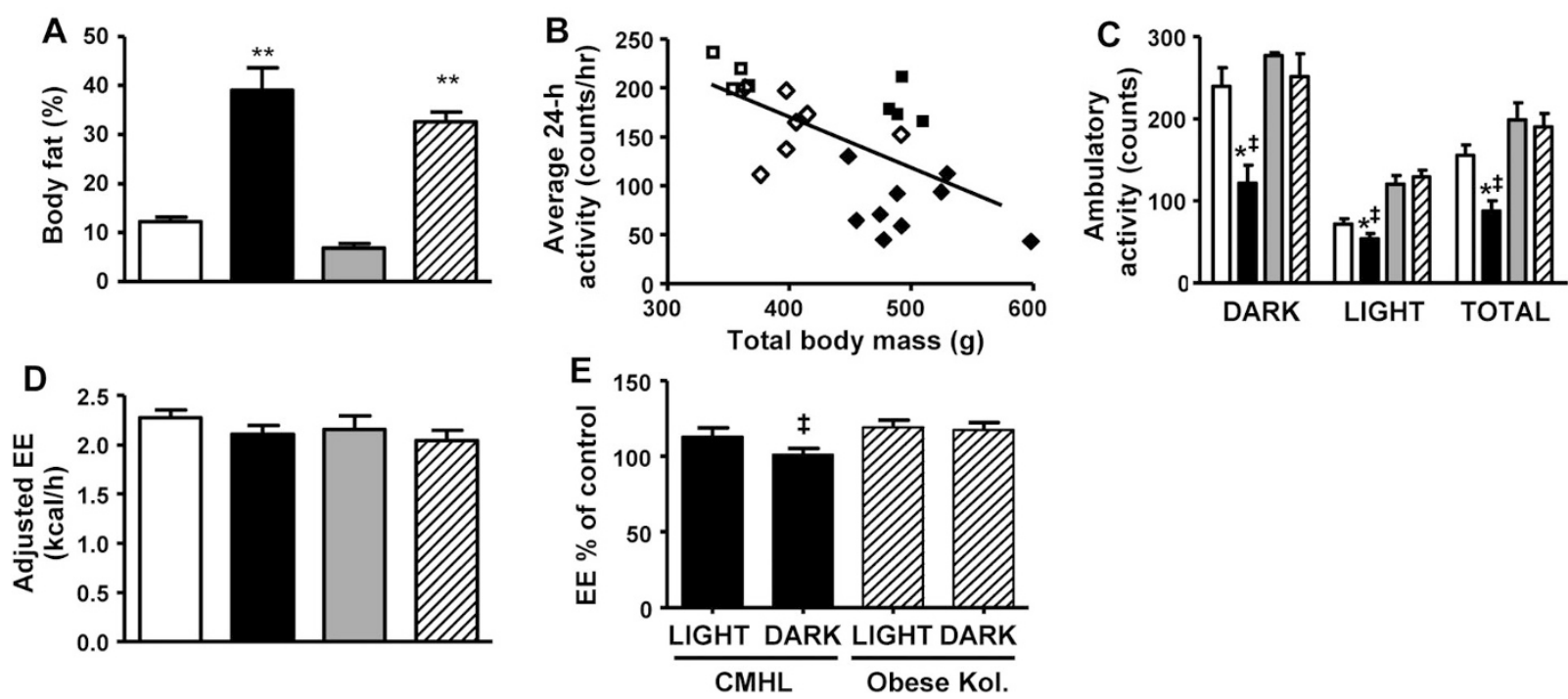

Figure 5. Body fat \% (A), average 24-h activity correlated with total body mass $(B)$ and ambulatory activity during the light and dark cycle $(C)$, EE adjusted for lean body mass (LBM), fat mass (FM), and activity (D) in CMHL (black bars and black diamonds) rats relative to sham-treated controls (white bars and white diamonds), lean (gray bars and white squares) and obese (hatched bars and black squares) Koletsky rats. Unadjusted EE in CMHL normalized to sham-treated rats and obese Koletsky rats normalized to lean Koletsky rats $(E)$. ${ }^{*} p<0.05 v s$. sham-treated controls; $\ddagger p<0.05 v s$. obese Koletsky rats at same cycle.

creased in CMHL rats relative to rats with lesions of the ARC induced by MSG, with electrolytic lesions of the VMN, or with lesions of both the VMN and DMN, and these effects were accompanied by more pronounced hyperleptinemia inappropriate for the degree of adiposity. Moreover, similar to what is observed in obese CP patients (34), CMHL rats exhibited a selective reduction in plasma $\alpha$-MSH levels relative to the other groups, and were also characterized by reduced locomotor activity, even after adjusting for differences in body size. The CMHL model therefore mimics both the neuroanatomical and metabolic phenotype of obese CP patients better than any of three other hypothalamic lesion models. We therefore anticipate that the CMHL model will be useful in future studies aimed at testing the efficacy of pharmaceutical treatment of obesity in patients with CP.

Although hypothalamic obesity in patients with $\mathrm{CP}$ commonly involves damage to the ARC, our findings suggest that this type of lesion cannot completely account for every clinical feature typical of obese $\mathrm{CP}$ patients. We found that although rats with neonatal MSG-induced ARC lesions exhibited increased body adiposity and hyperleptinemia, these animals also had reduced food intake and stunted growth. In contrast to destruction of the ARC alone, lesions of the VMN cause an obese-hyperphagic phenotype (35) characterized by hyperinsulinemia (36) and reduced sympathetic activity $(37,38)$, resembling some of the clinical features of patients with CP. Obese CP patients are characterized by a pronounced hyperleptinemia (39), hyperinsulinemia, and insulin resistance compared with BMI- and sex-matched controls (33).

The ARC is a key hypothalamic area that contains two neuronal populations important in the regulation of energy homeostasis. One of these neuronal populations expresses the pro-opimelanocortin (POMC) precursor, which is cleaved to generate melanocortin peptides such as $\alpha$-MSH that inhibit food intake and stimulate EE. An adjacent neuronal subset expresses neuropeptide Y (NPY), as well as agouti-related peptide (AgRP), a melanocortin-4 receptor (MC4R) antagonist. In contrast to POMC neurons, NPY/AgRP neurons stimulate food intake and reduce EE (40). These effects on energy balance are mediated via several second-order hypothalamic targets that express both the MC4R and NPY receptors (41) and regulate autonomic outflow (42). Among key neuronal populations that respond to input from the ARC are those found in the PVN that suppress food intake, such as corticotropin-releasing factor, TSH-releasing hormone, and oxytocin (43-45). Consequently, as a result of lesions that also effect the ARC, these two key neuronal populations would not be able to receive input from key hormones including leptin, insulin, peptide YY, and ghrelin (33) among others and thereby activate downstream pathways that regulate food intake and EE.

As a consequence, CMHL rats exhibit a more pronounced hyperphagic, obese phenotype compared with rats with lesions to the VMN or VMN/DMN. Moreover, consistent with that observed in obese CP patients, CMHL rats exhibit reduced ambulatory activity levels that cannot be accounted for by obesity, as this was not observed in obese-matched, leptin receptor-deficient animals. These data also suggest that the reduced activity in CMHL rats cannot be explained by deficient leptin-receptor signaling. In addition, plasma $\alpha$-MSH levels are reduced in CMHL rats relative to animals with lesions to the VMN/DMN and they exhibit pronounced hyperinsulinemia and hyperleptinemia within the first week of surgery. These increased plasma leptin and insulin levels cannot be completely accounted for by changes in obesity but are probably attributed to, at least in part, reduced sensing of these hormones due to the destruction of the ARC. Taken together, these data provide support that the CMHL model 
mimics the metabolic and neuroanatomical disturbances characteristic of obese $\mathrm{CP}$ patients and provides further evidence that the ARC is a critical brain area involved in the regulation of feeding and metabolism.

One of the major challenges ahead is to find an effective method for the treatment of hypothalamic obesity. Pharmaceutical approaches aimed at targeting neuronal populations in the ARC and/or VMN, such as leptin or ghrelin receptor antagonists, are unlikely to be effective due to the damage that occurs in these areas. A more promising and effective pharmaceutical approach is to activate targets downstream of leptin signaling. One attractive target is the MC4R, as these receptors are expressed in the PVN and hindbrain (46), leptin-induced anorexia requires MC4R signaling (47), and central administration of MC4R agonists reduce food intake and body weight (48). However, weight-loss induced with single agents has been relatively modest, due to the number of redundant feeding pathways, and the activation of compensatory mechanisms geared to defend against weight loss, such as the induction of melanin concentrating hormone $(\mathrm{MCH})$ expression in the LHA to stimulate feeding. Thus, a combination-based therapy including melanocortin agonists, combined with a $\mathrm{MCH}$ receptor- 1 antagonist may be more effective in the treatment of hypothalamic obesity. Our novel rat model is therefore important to help facilitate translational research addressing this vexing yet unsolved clinical problem.

In summary, we have created a rat model that recapitulates the unique constellation of features seen in obese $\mathrm{CP}$ patients. We demonstrate that lesions that include the ARC, in addition to the VMN and/or DMN, are required to elicit this phenotype. The creation of this CMHL model will help shed light onto the underlying mechanisms of hypothalamic obesity commonly seen in patients with $\mathrm{CP}$ and will be used to treat and/or prevent hypothalamic obesity.

Acknowledgments. We thank Michael W. Schwartz, Diabetes and Obesity Center of Excellence, Seattle, for constructive ideas and comments while developing this rat model, and S.R. Ojeda, OR National Primate Research Center/Oregon, Portland, for supporting us with equipment and advices for establishing the lesion model.

\section{REFERENCES}

1. Muller HL 2008 Childhood craniopharyngioma. Recent advances in diagnosis, treatment and follow-up. Horm Res 69:193-202

2. Garrè ML, Cama A 2007 Craniopharyngioma: modern concepts in pathogenesis and treatment. Curr Opin Pediatr 19:471-479

3. Harz KJ, Muller HL, Waldeck E, Pudel V, Roth C 2003 Obesity in patients with craniopharyngioma: assessment of food intake and movement counts indicating physical activity. J Clin Endocrinol Metab 88:5227-5231

4. Roth CL, Hunneman DH, Gebhardt U, Stoffel-Wagner B, Reinehr T, Muller HL 2007 Reduced sympathetic metabolites in urine of obese patients with craniopharyngioma. Pediatr Res 61:496-501

5. Müller HL, Handwerker G, Gebhardt U, Faldum A, Emser A, Kolb R, Sorensen N 2006 Melatonin treatment in obese patients with childhood craniopharyngioma and increased daytime sleepiness. Cancer Causes Control 17:583-589

6. Imura H, Kato Y, Nakai Y 1987 Endocrine aspects of tumors arising from suprasellar, third ventricular regions. Prog Exp Tumor Res 30:313-324

7. Curtis J, Daneman D, Hoffman HJ, Ehrlich RM 1994 The endocrine outcome after surgical removal of craniopharyngiomas. Pediatr Neurosurg 21:24-27

8. Jordaan GP, Roberts MC, Emsley RA 1996 Serotonergic agents in the treatment of hypothalamic obesity syndrome: a case report. Int J Eat Disord 20:111-113
9. Danielsson P, Janson A, Norgren S, Marcus C 2007 Impact sibutramine therapy in children with hypothalamic obesity or obesity with aggravating syndromes. J Clin Endocrinol Metab 92:4101-4106

10. Mason PW, Krawiecki N, Meacham LR 2002 The use of dextroamphetamine to treat obesity and hyperphagia in children treated for craniopharyngioma. Arch Pediatr Adolesc Med 156:887-892

11. Skorzewska A, Lal S, Waserman J, Guyda H 1989 Abnormal food-seeking behavior after surgery for craniopharyngioma. Neuropsychobiology 21:17-20

12. Ismail D, O'Connell MA, Zacharin MR 2006 Dexamphetamine use for management of obesity and hypersomnolence following hypothalamic injury. J Pediatr Endocrinol Metab 19:129-134

13. Lustig RH, Hinds PS, Ringwald-Smith K, Christensen RK, Kaste SC, Schreiber RE, Rai SN, Lensing SY, Wu S, Xiong X 2003 Octreotide therapy of pediatric hypothalamic obesity: a double-blind, placebo-controlled trial. J Clin Endocrinol Metab 88:2586-2592

14. King BM 2006 The rise, fall, and resurrection of the ventromedial hypothalamus in the regulation of feeding behavior and body weight. Physiol Behav 87:221-244

15. Anand BK, Brobeck JR 1951 Localization of a "feeding center" in the hypothalamus of the rat. Proc Soc Exp Biol Med 77:323-324

16. Coons EE, Levak M, Miller NE 1965 Lateral hypothalamus: learning of foodseeking response motivated by electrical stimulation. Science 150:1320-1321

17. Schwartz MW, Woods SC, Porte D, Seeley RJ, Baskin DG 2000 Central nervous system control of food intake. Nature 404:661-671

18. Schoelch C, Hubschle T, Schmidt I, Nuesslein-Hildesheim B 2002 MSG lesions decrease body mass of suckling-age rats by attenuating circadian decreases of energy expenditure. Am J Physiol Endocrinol Metab 283:E604-E611

19. Dolnikoff M, Martin-Hidalgo A, Machado UF, Lima FB, Herrera E 2001 Decreased lipolysis and enhanced glycerol and glucose utilization by adipose tissue prior to development of obesity in monosodium glutamate (MSG) treated-rats. Int J Obes Relat Metab Disord 25:426-433

20. Tokunaga K, Fukushima M, Kemnitz JW, Bray GA 1986 Effect of vagotomy on serum insulin in rats with paraventricular or ventromedial hypothalamic lesions. Endocrinology 119:1708-1711

21. Dube MG, Kalra PS, Crowley WR, Kalra SP 1995 Evidence of a physiological role for neuropeptide $\mathrm{Y}$ in ventromedial hypothalamic lesion-induced hyperphagia. Brain Res 690:275-278

22. Choi S, Sparks R, Clay M, Dallman MF 1999 Rats with hypothalamic obesity are insensitive to central leptin injections. Endocrinology 140:4426-4433

23. Paxinos G, Watson G 1986 The Rat Brain in Stereotaxic Coordinates. 2nd ed. Academic Press, New York, pp 43-188

24. Tokunaga K, Fukushima M, Kemnitz JW, Bray GA 1986 Comparison of ventromedial and paraventricular lesions in rats that become obese. Am J Physiol 251:R1221-R1227

25. Gelling RW, Al-Noori S, Pardini A, Morton GJ, Ogimoto K, Schwartz MW, Dempsey PJ 2008 Deficiency of TNFalpha converting enzyme (TACE/ADAM17) causes a lean, hypermetabolic phenotype in mice. Endocrinology 149:6053-6064

26. Kaiyala KJ, Morton GJ, Leroux BG, Ogimoto K, Wisse B, Schwartz MW 2010 Identification of body fat mass as a major determinant of metabolic rate in mice. Diabetes 59:1657-1666

27. Blevins JE, Stanley BG, Reidelberger RD 2000 Brain regions where cholecystokinin suppresses feeding in rats. Brain Res 860:1-10

28. Bernardis LL, Patterson BD 1968 Correlation between 'Lee index' and carcass fat content in weanling and adult female rats with hypothalamic lesions. J Endocrinol 40:527-528

29. Broberger C, Johansen J, Johansson C, Schalling M, Hokfelt T 1998 The neuropeptide Y/agouti gene-related protein (AGRP) brain circuitry in normal, anorectic, and monosodium glutamate-treated mice. Proc Natl Acad Sci USA 95:15043-15048

30. Dawson R, Wallace DR, Gabriel SM 1989 A pharmacological analysis of food intake regulation in rats treated neonatally with monosodium L-glutamate (MSG). Pharmacol Biochem Behav 32:391-398

31. de Souza CT, Nunes WM, Gobatto CA, de Mello MA 2003 Insulin secretion in monosodium glutamate (MSG) obese rats submitted to aerobic exercise training. Physiol Chem Phys Med NMR 35:43-53

32. Crawley JN, Kiss JZ 1985 Paraventricular nucleus lesions abolish the inhibition of feeding induced by systemic cholecystokinin. Peptides 6:927-935

33. Roth CL, Gebhardt U, Muller HL 2010 Appetite-regulating hormone changes in patients with craniopharyngioma. Obesity (Silver Spring), 2010 Apr 8. [Epub ahead of print]

34. Roth CL, Enriori PJ, Gebhardt U, Hinney A, Muller HL, Hebebrand J, Reinehr T, Cowley MA 2010 Changes of peripheral alpha-melanocyte-stimulating hormone in childhood obesity. Metabolism 59:186-194

35. Brooks CM, Lambert EF 1946 A study of the effect of limitation of food intake and the method of feeding on the rate of weight gain during hypothalamic obesity in the albino rat. Am J Physiol 147:695-707

36. Lustig RH, Post SR, Srivannaboon K, Rose SR, Danish RK, Burghen GA, Xiong X, Wu S, Merchant TE 2003 Risk factors for the development of obesity in children surviving brain tumors. J Clin Endocrinol Metab 88:611-616

37. Yoshimatsu H, Niijima A, Oomura Y, Yamabe K, Katafuchi T 1984 Effects of hypothalamic lesion on pancreatic autonomic nerve activity in the rat. Brain Res 303:147-152

38. Niijima A, Rohner-Jeanrenaud F, Jeanrenaud B 1984 Role of ventromedial hypothalamus on sympathetic efferents of brown adipose tissue. Am J Physiol 247:R650R654

39. Roth C, Wilken B, Hanefeld F, Schroter W, Leonhardt U 1998 Hyperphagia in children with craniopharyngioma is associated with hyperleptinaemia and a failure in the downregulation of appetite. Eur J Endocrinol 138:89-91 
40. Morton GJ, Schwartz MW 2001 The NPY/AgRP neuron and energy homeostasis. Int J Obes Relat Metab Disord 25:S56-S62

41. Liu H, Kishi T, Roseberry AG, Cai X, Lee CE, Montez JM, Friedman JM, Elmquist JK 2003 Transgenic mice expressing green fluorescent protein under the control of the melanocortin-4 receptor promoter. J Neurosci 23:7143-7154

42. Song CK, Bartness TJ 2001 CNS sympathetic outflow neurons to white fat that express MEL receptors may mediate seasonal adiposity. Am J Physiol Regul Integr Comp Physiol 281:R666-R672

43. Blevins JE, Morton GJ, Williams DL, Caldwell DW, Bastian LS, Wisse BE, Schwartz MW, Baskin DG 2009 Forebrain melanocortin signaling enhances the hindbrain satiety response to CCK-8. Am J Physiol Regul Integr Comp Physiol 296:R476-R484
44. Elmquist JK 2001 Hypothalamic pathways underlying the endocrine, autonomic, and behavioral effects of leptin. Int J Obes Relat Metab Disord 25:S78-S82

45. Harris M, Aschkenasi C, Elias CF, Chandrankunnel A, Nillni EA, Bjoorbaek C, Elmquist JK, Flier JS, Hollenberg AN 2001 Transcriptional regulation of the thyrotropin-releasing hormone gene by leptin and melanocortin signaling. J Clin Invest 107:111-120

46. Kishi T, Aschkenasi CJ, Lee CE, Mountjoy KG, Saper CB, Elmquist JK 2003 Expression of melanocortin 4 receptor mRNA in the central nervous system of the rat. J Comp Neurol 457:213-235

47. Seeley RJ, Yagaloff KA, Fisher SL, Burn P, Thiele TE, van Dijk G, Baskin DG, Schwartz MW 1997 Melanocortin receptors in leptin effects. Nature 390:349

48. Fan W, Boston BA, Kesterson RA, Hruby VJ, Cone RD 1997 Role of melanocortinergic neurons in feeding and the agouti obesity syndrome. Nature 385:165-168 